\title{
First Stereoselective Total Synthesis of Tumonoic Acid A and its Derivatives
}

\section{G. Nagalatha}

Siva Ganesh Narala

A. Venkat Narsaiah*

Organic Synthesis Laboratory, Fluoro-Agrochemicals Department, CSIR-Indian Institute of Chemical Technology, Hyderabad, 500007, Telangana, India

vnakkirala2001@yahoo.com

vnakkirala@csiriict.in

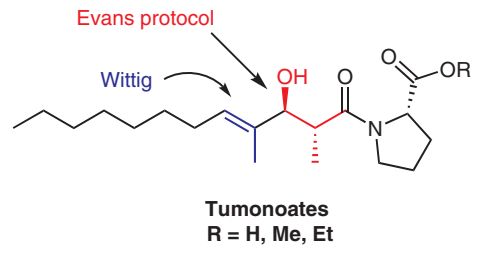

ria margaritifera, Lyngbya majuscule and Schizothrix calcicola (Figure 1). ${ }^{3}$ Ethyl tumonoate A exhibits anti-inflammatory activity in murine macrophage cells, inhibitory activity of calcium oscillations in neocortical neurons and in vitro anti-inflammatory activity in the RAW 264.7 murine macrophage cell-based nitric oxide assay with an $\mathrm{IC}_{50}$ of $9.8 \mu \mathrm{M}$ $(3.6 \mu \mathrm{g} / \mathrm{mL}){ }^{4}$ Ethyl tumonoate A shares a structural resemblance to a number of other cyanobacterial secondary metabolites, such as the viridamides and the microcolins. These molecules are composed of 8-12 carbon fatty acid chains connected to amino acid moieties.

Retrosynthetic analysis (Scheme 1) shows that ethyl tumonoate A could be derived from the intermediate, $(S)$ 4-benzyl-3-[(2R,3S,E)-3-hydroxy-2,4-dimethyldodec-4enoyl]oxazolidindin-2-one (8), which could, in turn, be obtained from $n$-octanal 2 through the Evans protocol without using protecting groups.

By using a Wittig protocol, octanal 2 underwent olefination with (carbethoxyethylidene)triphenyl phosphorane to give unsaturated ester 4 in $85 \%$ yield, ${ }^{5}$ which was then reduced to the allylic alcohol $\mathbf{5}$ in $92 \%$ yield by treating with phyletic bacterial phylum that are extraordinarily rich in bioactive molecules. Tumonoic acids $\mathrm{A}-\mathrm{C}$ were isolated from the marine cyanobacteria (blue-green algae) Oscillato-

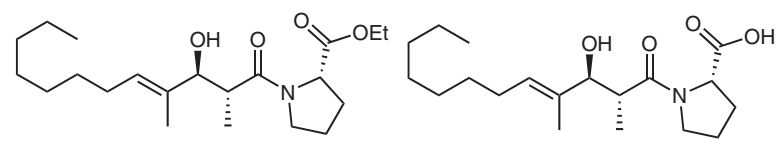

Ethyl tumonoate A (1a)

Tumonoic acid A (1b)

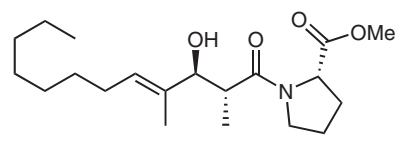

Methyl tumonoate A (1c)

Figure 1

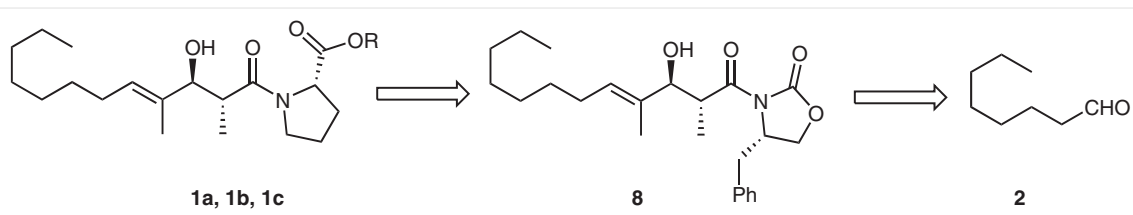

Scheme 1 
DIBAL-H (Scheme 2). Subsequent oxidation of alcohol 5 with $\mathrm{MnO}_{2}$ in anhydrous hexane provided the corresponding aldehyde 6 in $97 \%$ yield. ${ }^{6}$

The (E)-2-methyldec-2-enal 6 was subjected to diastereoselective aldol reaction by following the Evans protocol, ${ }^{7}$ catalysed by magnesium chloride, using (S)-4-benzyl-3propionyloxazolidin-2-one $\mathbf{7}$ as the chiral auxiliary to give anti-aldol product 8 in $67 \%$ yield, with excellent diastereoselectivity, as determined by ${ }^{1} \mathrm{H}$ NMR spectroscopy. The removal of the chiral auxiliary was achieved by oxidative hydrolysis with $\mathrm{LiOH}, \mathrm{H}_{2} \mathrm{O}_{2}$ in THF- $\mathrm{H}_{2} \mathrm{O}$, furnishing the desired (2R,3S,E)-3-hydroxy-2,4-dimethyldodec-4-enoic acid (9). ${ }^{8}$ Finally, amide bond formation was achieved by couplingacid $\mathbf{9}$ with L-proline ester in the presence of EDC.HCl and HOBt to give the target molecule, ethyl tumonoate A 1a, as a yellow liquid in $82 \%$ yield, ${ }^{9}$ with a specific rotation $[\alpha]_{D}{ }^{25}=-78$ $\left(c=0.4, \mathrm{CHCl}_{3}\right)\left\{\mathrm{Lit}^{4}{ }^{4}[\alpha]_{\mathrm{D}}{ }^{25}=-77.5\left(\mathrm{c}=1, \mathrm{CHCl}_{3}\right)\right\}$. Tumonoic acid $\mathrm{A}(\mathbf{1 b})$ was obtained as a pale-yellow liquid with a specific rotation $[\alpha]_{D}{ }_{D}^{25}=-80\left(\mathrm{c}=0.7, \mathrm{CHCl}_{3}\right)\left\{\mathrm{Lit}^{3}[\alpha]_{D}{ }^{25}=-79\right.$ $\left(\mathrm{c}=1.1, \mathrm{CHCl}_{3}\right)$ \} by hydrolysis of ethyl tumonoate A using $\mathrm{EtOH}$ and $\mathrm{NaOH}$. Methyl tumonoate $\mathrm{A}(\mathbf{1 c})$ with specific rotation $[\alpha]_{D}^{25}=-52\left(\mathrm{c}=1, \mathrm{CHCl}_{3}\right)\left\{\mathrm{Lit}^{3}[\alpha]_{D}^{25}=-51(\mathrm{c}=1.3\right.$,
$\left.\left.\mathrm{CHCl}_{3}\right)\right\}$ was obtained by esterification of tumonoic acid A (1b) using methyl iodide and potassium carbonate in DMF. The structures of all the products were confirmed by their spectroscopic data and by comparison with reported data.

In conclusion, we have accomplished the stereoselective total synthesis of naturally occurring tumonoic acid A and its derivatives, starting from $n$-octanal, without using any protection protocols. The synthetic strategy involves successful application of the magnesium chloride-catalysed anti-aldol reaction under the Evans protocol and amide coupling.

All air- and moisture-sensitive reactions were carried out under an inert atmosphere (nitrogen or argon). Oven-dried glass apparatus was used to perform all reactions. Freshly distilled anhydrous solvents were used for air- and moisture-sensitive reactions. Commercially available reagents were used as purchased. Purification of compounds was carried out by column chromatography, using silica gel (60-120 mesh). ${ }^{1} \mathrm{H}$ NMR and ${ }^{13} \mathrm{C}$ NMR spectra were recorded in $\mathrm{CDCl}_{3}$ at 300 MHz, Bruker Avance II and $500 \mathrm{MHz}$, Bruker Avance III HD, using TMS as an internal standard. IR spectra were recorded with a Perkin-Elmer FTIR 240-c spectrophotometer using $\mathrm{KBr} /$ thin-film optics. Mass spectra were recorded with a Finnigan MAT 1020 mass spectrometer

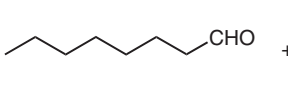

2<smiles>CCCCCCCC=C(C)CO</smiles>

5<smiles>CCCCCCC/C=C(\C)[C@@H](O)[C@H](C)C(=O)N1C(=O)OC[C@H]1Cc1ccccc1</smiles><smiles>CCCCCCC/C=C(\C)[C@@H](O)[C@@H](C)C(=O)N1CCC[C@H]1C(=O)OCC</smiles>

Ethyl tumonoate A (1a)<smiles>CCCCCCC/C=C(\C)[C@@H](O)[C@H](C)C(=O)N1CCC[C@H]1C(=O)OC</smiles>

Methyl tumonoate A (1c)

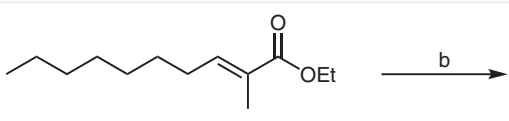

4<smiles>CCC(=O)N1C(=O)OC[C@H]1Cc1ccccc1</smiles>

6<smiles>CCCCCCC/C=C(\C)[C@@H](O)[C@@H](C)C(=O)O</smiles><smiles>CCCCCCC/C=C(\C)[C@@H](O)[C@@H](C)C(=O)N1CCC[C@H]1C(=O)O</smiles>

Tumonoic acid A (1b)

Scheme 2 Reagents and conditions: (a) Benzene, reflux, $3 \mathrm{~h}, 85 \%$; (b) DIBAL-H, $-78{ }^{\circ} \mathrm{C}, \mathrm{CH}_{2} \mathrm{Cl}_{2}, 30$ min, $92 \%$; (c) MnO , anhydrous hexane, $3 \mathrm{~h}, 97 \%$; (d) (i) $\mathrm{MgCl}_{2}$, TMS-Cl, Et ${ }_{3} \mathrm{~N}$, EtOAc, 24 h; (ii) p-TsOH, MeOH, 67\%; (e) LiOH, $\mathrm{H}_{2} \mathrm{O}_{2}$, THF- $\mathrm{H}_{2} \mathrm{O}(4: 1)$, $\mathrm{Na}_{2} \mathrm{SO}_{3}, 2$ h, 75\%; (f) (S)-ethyl pyrrolidine-2-carboxylate, EDC. $\mathrm{HCl}, 1$-hydroxybenzotriazole (HOBt), $\mathrm{CH}_{2} \mathrm{Cl}_{2}$, Et ${ }_{3} \mathrm{~N}, 1 \mathrm{~h}, 82 \%$; (g) NaOH, MeOH-THF (1:1), 1 h, $80 \%$; (h) $\mathrm{K}_{2} \mathrm{CO}_{3}, \mathrm{CH}_{3} \mathrm{l}, \mathrm{DMF}, 90{ }^{\circ} \mathrm{C}, 2 \mathrm{~h}, 78 \%$. 
operating at $70 \mathrm{eV}$. Specific rotation values were recorded with a Horiba sepa 300 polarimeter. High-resolution mass spectra (HRMS) [ESI+] were obtained with an Orbitrap, Thermo Scientific.

\section{(E)-Ethyl 2-methyldec-2-enoate (4)}

To a stirred solution of $n$-octanal $(2.00 \mathrm{~g}, 15.6 \mathrm{mmol})$ in benzene ( 30 $\mathrm{mL}$ ) was added (carbethoxy ethylidene)triphenyl phosphorane (8.50 $\mathrm{g}, 27.0 \mathrm{mmol}$ ) and the reaction mixture was heated to reflux for $3 \mathrm{~h}$. After completion of reaction, the solvent was removed under reduced pressure and the crude product was purified by column chromatography, eluting with EtOAc-hexane (5:95). The pure product 4 (2.80 g, $85 \%$ ) was obtained as a yellow liquid.

IR (neat): 2851, 1711, 1639, 1549, 1462, 1449, 1377, 1216, 1093, 771 $\mathrm{cm}^{-1}$.

${ }^{1} \mathrm{H} \mathrm{NMR}\left(300 \mathrm{MHz}, \mathrm{CDCl}_{3}\right): \delta=6.80-6.72(\mathrm{~m}, 1 \mathrm{H}$ ) $), 4.19(\mathrm{q}, J=7.0 \mathrm{~Hz}$, $2 \mathrm{H}), 2.16$ (q, J = 7.2 Hz, $2 \mathrm{H}), 1.83(\mathrm{~s}, 3 \mathrm{H}), 1.38-1.20(\mathrm{~m}, 13 \mathrm{H}), 0.88(\mathrm{t}$, $J=6.7 \mathrm{~Hz}, 3 \mathrm{H})$.

${ }^{13} \mathrm{C}$ NMR $\left(75 \mathrm{MHz}, \mathrm{CDCl}_{3}\right): \delta=168.2,142.3,127.5,60.2,31.7,29.3$, 29.0, 28.6, 28.5, 22.5, 14.2, 14.0, 12.2 .

MS (ESI): $m / z=213[\mathrm{M}+\mathrm{H}] .^{+}$

\section{(E)-2-Methyldec-2-en-1-ol (5)}

To a stirred solution of compound $4(2.30 \mathrm{~g}, 10.8 \mathrm{mmol})$ in anhydrous $\mathrm{CH}_{2} \mathrm{Cl}_{2}$ (30 mL), DIBAL-H (1 M in toluene, $23.7 \mathrm{~mL}, 23.7 \mathrm{mmol}$ ) was added at $-78{ }^{\circ} \mathrm{C}$. After $30 \mathrm{~min}$, the reaction mixture was quenched with $\mathrm{MeOH}(1 \mathrm{~mL})$ and a saturated solution of Rochelle's salt $(50 \mathrm{~mL})$ and allowed to stir for $1 \mathrm{~h}$. The mixture was extracted with $\mathrm{CH}_{2} \mathrm{Cl}_{2}$ $(2 \times 30 \mathrm{~mL})$, washed with brine, dried over $\mathrm{Na}_{2} \mathrm{SO}_{4}$, filtered, and concentrated under reduced pressure. The residue was purified by column chromatography, eluting with EtOAc-hexane (1:9) mixture to furnish compound $\mathbf{5}(1.70 \mathrm{~g}, 92 \%)$ as a yellow liquid.

IR (neat): 3450, 2924, 2854, 1601, 1641, 1498, 1460, 1260, 1175 , $1023,756 \mathrm{~cm}^{-1}$.

${ }^{1} \mathrm{H} \mathrm{NMR}\left(300 \mathrm{MHz}, \mathrm{CDCl}_{3}\right): \delta=5.41(\mathrm{t}, J=6.9 \mathrm{~Hz}, 1 \mathrm{H}), 4.00(\mathrm{~s}, 2 \mathrm{H})$, 2.09-1.97 (m, $2 \mathrm{H}), 1.67(\mathrm{~s}, 3 \mathrm{H}), 1.38-1.20(\mathrm{~m}, 10 \mathrm{H}), 0.88(\mathrm{t}, J=$ $6.5 \mathrm{~Hz}, 3 \mathrm{H})$.

${ }^{13} \mathrm{C} \mathrm{NMR}\left(75 \mathrm{MHz}, \mathrm{CDCl}_{3}\right): \delta=134.4,126.3,68.7,31.7,29.4,29.2,29.1$, 27.5, 22.5, 13.9, 13.4 .

MS (ESI): $m / z=171[\mathrm{M}+\mathrm{H}] .^{+}$

\section{(E)-2-Methyldec-2-enal (6)}

To a stirred solution of compound $\mathbf{5}(1.60 \mathrm{~g}, 9.4 \mathrm{mmol})$ in anhydrous hexane $(20 \mathrm{~mL})$ was added activated $\mathrm{MnO}_{2}(8.20 \mathrm{~g}, 94 \mathrm{mmol})$. After stirring at r.t. for $3 \mathrm{~h}$, the reaction mixture was filtered through a bed of Celite ${ }^{\circledR}$. The filtrate was concentrated under reduced pressure and the residue was purified by column chromatography, eluting with EtOAc-hexane (5:95) mixture to afford 6 (1.53 g, 97\%) as a pale-yellow liquid.

IR (neat): 2923, 2853, 1689, 1644, 1461, 1419, 1379, 1279, 1219, 933 , $772 \mathrm{~cm}^{-1}$.

${ }^{1} \mathrm{H}$ NMR $\left(300 \mathrm{MHz}, \mathrm{CDCl}_{3}\right): \delta=9.40(\mathrm{~s}, 1 \mathrm{H}), 6.50(\mathrm{t}, J=7.3 \mathrm{~Hz}, 1 \mathrm{H})$, $2.35(\mathrm{q}, J=7.2 \mathrm{~Hz}, 2 \mathrm{H}), 1.75(\mathrm{~s}, 3 \mathrm{H}), 1.38-1.20(\mathrm{~m}, 10 \mathrm{H}), 0.88(\mathrm{t}, J=$ $6.7 \mathrm{~Hz}, 3 \mathrm{H})$.

${ }^{13} \mathrm{C}$ NMR $\left(75 \mathrm{MHz}, \mathrm{CDCl}_{3}\right): \delta=195.4,155.1,139.2,31.7,29.2,29.0$, 28.9, 28.3, 22.6, 14.0, 9.1.

MS (ESI): $m / z=169[\mathrm{M}+\mathrm{H}] .^{+}$
(S)-4-Benzyl-3-[(2R,3S,E)-3-hydroxy-2,4-dimethyldodec-4enoyl]oxazolidin-2-one (8)

To a stirred solution of (S)-4-benzyl-3-propionyloxazolidin-2-one 7 $(2.08 \mathrm{~g}, 11.8 \mathrm{mmol})$ in anhydrous EtOAc $(20 \mathrm{~mL})$ was added anhydrous $\mathrm{MgCl}_{2}(0.17 \mathrm{~g}, 1.7 \mathrm{mmol})$, triethylamine (1.6 mL, $\left.11.8 \mathrm{mmol}\right)$ and trimethylsilyl chloride $(1.12 \mathrm{~mL}, 9.0 \mathrm{mmol})$ sequentially. The reaction mixture was stirred for $10 \mathrm{~min}$ and then aldehyde $\mathbf{6}(1 \mathrm{~g}, 5.9$ $\mathrm{mmol})$, dissolved in anhydrous EtOAc $(10.0 \mathrm{~mL})$, was added to the reaction mixture. After $24 \mathrm{~h}$, the reaction mixture was filtered through a plug of silica-gel using EtOAc as the eluent. The solvent was removed in vacuo, then $\mathrm{MeOH}(20 \mathrm{~mL})$ was added to the residue, along with $p$-TsOH (1.69 g, $8.8 \mathrm{mmol}$ ). After $15 \mathrm{~min}$, desilylation was complete as monitored by TLC. After concentration in vacuo the desilylated mixture was purified by column chromatography, eluting with EtOAc-hexane (2:8). The pure product $8(1.20 \mathrm{~g}, 67 \%)$ was obtained as a yellow liquid, with 19:1 diastereoselectivity.

$[\alpha]_{\mathrm{D}}^{25}=+20\left(\mathrm{c}=0.6, \mathrm{CHCl}_{3}\right)$.

IR (neat): 3451, 2924, 2853, 1780, 1636, 1458, 1385, 1261, 1103 , $1016,926,853 \mathrm{~cm}^{-1}$.

${ }^{1} \mathrm{H}$ NMR $\left(300 \mathrm{MHz}, \mathrm{CDCl}_{3}\right): \delta=7.38-7.20(\mathrm{~m}, 5 \mathrm{H}, \mathrm{ArH}), 5.49(\mathrm{t}, J=$ $6.9 \mathrm{~Hz}, 1 \mathrm{H}), 4.70(\mathrm{ddd}, J=10.1,6.9,3.3 \mathrm{~Hz}, 1 \mathrm{H}), 4.27-4.00(\mathrm{~m}, 4 \mathrm{H})$, 3.32 (dd, $J=13.4,3.2 \mathrm{~Hz}, 1 \mathrm{H}), 2.84-2.74(\mathrm{~m}, 1 \mathrm{H}), 2.10-1.99(\mathrm{~m}, 2 \mathrm{H})$, $1.68(\mathrm{~s}, 3 \mathrm{H}), 1.38-1.20(\mathrm{~m}, 10 \mathrm{H}), 1.06(\mathrm{~d}, J=6.4 \mathrm{~Hz}, 3 \mathrm{H}), 0.88(\mathrm{t}, J=$ $6.6 \mathrm{~Hz}, 3 \mathrm{H})$.

${ }^{13} \mathrm{C} \mathrm{NMR}\left(75 \mathrm{MHz}, \mathrm{CDCl}_{3}\right): \delta=176.7,153.8,135.3,134.1,130.1,129.4$, 128.9, 127.2, 81.4, 66.0, 55.6, 40.6, 37.7, 31.8, 29.6, 29.3, 29.1, 27.6, 22.6, 14.7, 14.0, 10.7 .

MS (ESI): $m / z=424[\mathrm{M}+\mathrm{Na}]^{+}$.

HRMS (ESI): $m / z[\mathrm{M}+\mathrm{H}]^{+}$calcd for $\mathrm{C}_{24} \mathrm{H}_{36} \mathrm{O}_{4} \mathrm{~N}$ : 402.26389; found: 402.26404 .

\section{(2R,3S,E)-3-Hydroxy-2,4-dimethyldodec-4-enoic Acid (9)}

To a stirred solution of compound $8(1.10 \mathrm{~g}, 2.7 \mathrm{mmol})$ in THF- $\mathrm{H}_{2} \mathrm{O}$ $(4: 1,18 \mathrm{~mL})$ at $0{ }^{\circ} \mathrm{C}$ was added aqueous $\mathrm{H}_{2} \mathrm{O}_{2}(30 \%, 1.86 \mathrm{~mL}, 16.4$ $\mathrm{mmol})$ followed by $\mathrm{LiOH} \cdot \mathrm{H}_{2} \mathrm{O}(0.34 \mathrm{~g}, 8.2 \mathrm{mmol})$. The reaction mixture was then warmed to r.t. and stirred for $2 \mathrm{~h}$. After completion of reaction, as monitored by TLC, the excess peroxide was quenched with $\mathrm{Na}_{2} \mathrm{SO}_{3}$ solution ( $1 \mathrm{M}, 10.90 \mathrm{~mL}$ ) and the mixture was stirred for an additional $20 \mathrm{~min}$. The solvent was removed under reduced pressure and the chiral auxiliary was recovered by $\mathrm{CH}_{2} \mathrm{Cl}_{2}$ extraction $(3 \times 10$ $\mathrm{mL})$. The aqueous phase was acidified with $\mathrm{HCl}(1 \mathrm{M})$ to $\mathrm{pH} 2$ and then extracted with EtOAc $(3 \times 15 \mathrm{~mL})$. The combined organic layers were washed with brine, dried over $\mathrm{Na}_{2} \mathrm{SO}_{4}$, filtered, and concentrated in vacuo. The crude product was purified by column chromatography, eluting with EtOAc-hexane (4:6) to afford pure $\mathbf{9}(0.50 \mathrm{~g}, 75 \%)$ as a pale-yellow liquid.

$[\alpha]_{\mathrm{D}}^{25}=-23\left(\mathrm{c}=0.8, \mathrm{CHCl}_{3}\right)$.

IR (neat): 3445, 2924, 2854, 1550, 1713, 1459, 1379, 1258, 1219, $1200,1009,915,848,771 \mathrm{~cm}^{-1}$.

${ }^{1} \mathrm{H}$ NMR $\left(300 \mathrm{MHz}, \mathrm{CDCl}_{3}\right): \delta=5.46(\mathrm{t}, J=7.0 \mathrm{~Hz}, 1 \mathrm{H}), 4.12(\mathrm{~d}, J=$ $9.4 \mathrm{~Hz}, 1 \mathrm{H}), 2.74-2.60(\mathrm{~m}, 1 \mathrm{H}), 2.04(\mathrm{q}, J=6.8 \mathrm{~Hz}, 2 \mathrm{H}), 1.61(\mathrm{~s}, 3 \mathrm{H})$, $1.38-1.20(\mathrm{~m}, 10 \mathrm{H}), 1.05(\mathrm{~d}, J=6.9 \mathrm{~Hz}, 3 \mathrm{H}), 0.88(\mathrm{t}, J=6.6 \mathrm{~Hz}, 3 \mathrm{H})$.

${ }^{13} \mathrm{C}$ NMR $\left(75 \mathrm{MHz}, \mathrm{CDCl}_{3}\right): \delta=180.1,133.4,130.8,80.1,42.9,31.8$, 29.6, 29.2, 29.1, 27.5, 22.6, 14.1, 14.0, 10.4 .

MS (ESI): $m / z=265[\mathrm{M}+\mathrm{Na}]^{+}$.

HRMS (ESI): $m / z[\mathrm{M}+\mathrm{Na}]^{+}$calcd for $\mathrm{C}_{14} \mathrm{H}_{26} \mathrm{O}_{3} \mathrm{Na}$ : 265.17742; found: 265.17751. 
(S)-Ethyl-1-[(2R,3S,E)-3-hydroxy-2,4-dimethyldodec-4-enoyl]-pyrolidine-2-carboxylate (Ethyl tumonoate $\mathrm{A} ; \mathrm{1a}$ )

To a stirred solution of $\mathbf{9}(0.40 \mathrm{~g}, 1.6 \mathrm{mmol})$ in anhydrous $\mathrm{CH}_{2} \mathrm{Cl}_{2}(5$ $\mathrm{mL})$ was added EDC. $\mathrm{HCl}(0.35 \mathrm{~g}, 1.8 \mathrm{mmol})$ and $\mathrm{HOBt}(0.25 \mathrm{~g}, 1.8$ $\mathrm{mmol}$ ). After $10 \mathrm{~min}$, a mixture of (S)-ethyl pyrrolidine-2-carboxylate $(0.28 \mathrm{~g}, 1.9 \mathrm{mmol})$ and triethylamine $(0.69 \mathrm{~mL}, 4.9 \mathrm{mmol})$ in $\mathrm{CH}_{2} \mathrm{Cl}_{2}(2$ $\mathrm{mL}$ ) was added dropwise, and the reaction mixture was stirred for $1 \mathrm{~h}$. After completion of the reaction, as monitored by TLC, the mixture was diluted with $\mathrm{CH}_{2} \mathrm{Cl}_{2}(60 \mathrm{~mL})$ and water $(20 \mathrm{~mL})$. The organic layer was separated and washed with $\mathrm{HCl}(1 \mathrm{M}, 5 \mathrm{~mL})$, water $(15 \mathrm{~mL})$, saturated aqueous $\mathrm{NaHCO}_{3}(10 \mathrm{~mL})$ and brine $(15 \mathrm{~mL})$. The organic layer was dried over $\mathrm{Na}_{2} \mathrm{SO}_{4}$, filtered, and concentrated under reduced pressure. The crude product was purified by column chromatography, eluting with EtOAc-hexane (3:7) mixture to afford the pure product 1a $(0.5 \mathrm{~g}, 82 \%)$ as a yellowish liquid.

$[\alpha]_{\mathrm{D}}^{25}=-78\left(\mathrm{c}=0.4, \mathrm{CHCl}_{3}\right)^{4}$

IR (neat): 3420, 2925, 2854, 1743, 1626, 1565, 1461, 1373, 1274, $1187,1095,1024,917,754,703 \mathrm{~cm}^{-1}$.

${ }^{1} \mathrm{H}$ NMR $\left(500 \mathrm{MHz}, \mathrm{CDCl}_{3}\right): \delta=5.44(\mathrm{t}, J=7.0 \mathrm{~Hz}, 1 \mathrm{H}), 4.50(\mathrm{dd}, J=8.6$, $4.2 \mathrm{~Hz}, 1 \mathrm{H}), 4.24-4.12(\mathrm{~m}, 3 \mathrm{H}), 3.65(\mathrm{t}, J=6.6 \mathrm{~Hz}, 2 \mathrm{H}), 2.75(\mathrm{p}, J=$ $7.1 \mathrm{~Hz}, 1 \mathrm{H}), 2.39-2.15(\mathrm{~m}, 3 \mathrm{H}), 2.10-1.94(\mathrm{~m}, 3 \mathrm{H}), 1.62(\mathrm{~s}, 3 \mathrm{H})$, 1.38-1.20 (m, $13 \mathrm{H}), 1.10$ (d, $J=7.0 \mathrm{~Hz}, 3 \mathrm{H}), 0.88(\mathrm{t}, J=6.9 \mathrm{~Hz}, 3 \mathrm{H})$.

${ }^{13} \mathrm{C}$ NMR $\left(125 \mathrm{MHz}, \mathrm{CDCl}_{3}\right): \delta=174.9,172.1,134.0,129.1,79.8,61.0$, 58.6, 47.0, 41.1, 31.8, 29.6, 29.4, 29.1, 29.0, 27.5, 24.7, 22.6, 14.4, 14.1, 11.4 .

MS (ESI): $m / z=368[\mathrm{M}+\mathrm{H}]^{+}$.

HRMS (ESI): $m / z[\mathrm{M}+\mathrm{H}]^{+}$calcd for $\mathrm{C}_{21} \mathrm{H}_{38} \mathrm{O}_{4} \mathrm{~N}$ : 368.27954; found: 368.27962 .

(S)-1-((2R,3S,E)-3-Hydroxy-2,4-dimethyldodec-4-enoyl)pyrrolidine-2-carboxylic Acid (Tumonoic acid A; 1b)

To a stirred solution of compound $\mathbf{1 a}(0.40 \mathrm{~g}, 1.08 \mathrm{mmol})$ in methanol-THF $(1: 1,8 \mathrm{~mL})$ aq. $\mathrm{NaOH}(2 \mathrm{M}, 1.6 \mathrm{~mL})$ was added and the reaction mixture was stirred at r.t. for $1 \mathrm{~h}$. After completion of the reaction, as monitored by TLC, the solvent was removed in vacuo. The residue was diluted with water $(2 \mathrm{~mL})$ and $\mathrm{HCl}(2 \mathrm{M}, 1.6 \mathrm{~mL})$ and the precipitated product was extracted with EtOAc $(2 \times 10 \mathrm{~mL})$. The combined organic layers were washed with brine, dried over $\mathrm{Na}_{2} \mathrm{SO}_{4}$, filtered and concentrated under reduced pressure. The crude product was purified by column chromatography, eluting with methanolchloroform (2:8) to furnish pure $\mathbf{1 b}(0.29 \mathrm{~g}, 80 \%)$ as a pale-yellow liquid.

$[\alpha]_{\mathrm{D}}{ }^{25}=-80\left(\mathrm{c}=0.7, \mathrm{CHCl}_{3}\right)^{3}$

IR (neat): 3418, 2924, 2855, 1731, 1618, 1458, 1262, 1217, 1192, $1017,927,771 \mathrm{~cm}^{-1}$.

${ }^{1} \mathrm{H}$ NMR $\left(500 \mathrm{MHz}, \mathrm{CDCl}_{3}\right): \delta=5.46(\mathrm{t}, J=7.0 \mathrm{~Hz}, 1 \mathrm{H}), 4.65-4.58(\mathrm{~m}$, $1 \mathrm{H}), 4.17(\mathrm{~d}, J=8.5 \mathrm{~Hz}, 1 \mathrm{H}), 3.75-3.66(\mathrm{~m}, 1 \mathrm{H}), 3.64-3.57(\mathrm{~m}, 1 \mathrm{H})$, $2.80(\mathrm{p}, J=7.3 \mathrm{~Hz}, 1 \mathrm{H}), 2.45-2.28(\mathrm{~m}, 2 \mathrm{H}), 2.07-1.99(\mathrm{~m}, 4 \mathrm{H}), 1.62(\mathrm{~s}$, $3 \mathrm{H}), 1.38-1.20(\mathrm{~m}, 10 \mathrm{H}), 1.03(\mathrm{~d}, J=6.8 \mathrm{~Hz}, 3 \mathrm{H}), 0.88(\mathrm{t}, J=6.8 \mathrm{~Hz}$, $3 \mathrm{H})$.

${ }^{13} \mathrm{C}$ NMR $\left(125 \mathrm{MHz}, \mathrm{CDCl}_{3}\right): \delta=177.8,177.6,133.7,130.3,80.4,60.3$, $47.9,41.3$, 31.8, 29.6, 29.2, 29.1, 27.6, 27.4, 24.7, 22.6, 14.3, 14.0, 10.9 . MS (ESI): $m / z=338[\mathrm{M}-\mathrm{H}]^{+}$.

HRMS (ESI): $m / z[M+H]^{+}$calcd for $\mathrm{C}_{19} \mathrm{H}_{34} \mathrm{O}_{4} \mathrm{~N}$ : 340.24824; found: 340.24841
(S)-Methyl-1-((2R,3S,E)-3-hydroxy-2,4-dimethyldodec-4enoyl)pyrrolidine-2-carboxylate (Methyl tumonoate A; 1c)

A stirred solution of tumonoic acid A $(0.20 \mathrm{~g}, 0.5 \mathrm{mmol})$ in DMF (3 $\mathrm{mL}$ ) was cooled to $0{ }^{\circ} \mathrm{C}$ and $\mathrm{K}_{2} \mathrm{CO}_{3}(0.13 \mathrm{~g}, 0.9 \mathrm{mmol})$ was added, followed by iodomethane $(0.04 \mathrm{~mL}, 0.7 \mathrm{mmol})$. The reaction mixture was allowed to warm to r.t. and stirred for $1 \mathrm{~h}$, when it became a yellow heterogeneous solution. The mixture was then heated to $90^{\circ} \mathrm{C}$ for $1 \mathrm{~h}$ and cooled to r.t. The reaction mixture was extracted with EtOAc $(2 \times 10 \mathrm{~mL})$ and the combined organic layers were washed with brine, dried over $\mathrm{Na}_{2} \mathrm{SO}_{4}$, filtered and concentrated under reduced pressure. The crude compound was purified by column chromatography, eluting with EtOAc-hexane (3:7). Pure product $1 \mathrm{c}(0.17 \mathrm{~g}$, $78 \%)$ was obtained as a yellow liquid.

$[\alpha]_{\mathrm{D}}^{25}=-52\left(\mathrm{c}=1, \mathrm{CHCl}_{3}\right)$. $^{3}$

IR (neat): 3449, 2924, 2853, 1744, 1629, 1459, 1169, $766 \mathrm{~cm}^{-1}$.

${ }^{1} \mathrm{H} \mathrm{NMR}\left(500 \mathrm{MHz}, \mathrm{CDCl}_{3}\right): \delta=5.44(\mathrm{t}, J=7.0 \mathrm{~Hz}, 1 \mathrm{H}), 4.52(\mathrm{dd}, J=8.7$, $4.1 \mathrm{~Hz}, 1 \mathrm{H}), 4.15(\mathrm{~d}, J=7.7 \mathrm{~Hz}, 1 \mathrm{H}), 3.73(\mathrm{~s}, 3 \mathrm{H}), 3.66(\mathrm{t}, J=6.6 \mathrm{~Hz}$, $2 \mathrm{H}), 2.76(\mathrm{p}, J=7.1 \mathrm{~Hz}, 1 \mathrm{H}), 2.38-2.28(\mathrm{~m}, 2 \mathrm{H}), 2.24-1.94(\mathrm{~m}, 4 \mathrm{H})$, $1.62(\mathrm{~s}, 3 \mathrm{H}), 1.36-1.20(\mathrm{~m}, 10 \mathrm{H}), 1.10(\mathrm{~d}, J=7.0 \mathrm{~Hz}, 3 \mathrm{H}), 0.88(\mathrm{t}, J=$ $6.8 \mathrm{~Hz}, 3 \mathrm{H})$.

${ }^{13} \mathrm{C}$ NMR $\left(125 \mathrm{MHz}, \mathrm{CDCl}_{3}\right): \delta=175.0,172.6,134.0,129.2,79.9,58.5$, 52.2, 47.0, 41.1, 31.9, 29.6, 29.4, 29.2, 29.0, 27.5, 24.7, 22.6, 14.3, 14.0, 11.4 .

MS (ESI): $m / z=354[\mathrm{M}+\mathrm{H}]^{+}$.

HRMS (ESI): $m / z$ [M $+\mathrm{H}]^{+}$calcd for $\mathrm{C}_{20} \mathrm{H}_{36} \mathrm{O}_{4} \mathrm{~N}: 354.26389$; found: 354.26431.

\section{Funding Information}

SGN and GNL are grateful to UGC-New Delhi and CSIR-New Delhi, respectively, for providing Fellowships, the Ministry of Science and Technology for providing financial support under DST-SERB-GAP0563 and the Director CSIR-IICT for providing facilities. IICT Commun. No: IICT/pubs./2018/268.

\section{Supporting Information}

Supporting information for this article is available online at https://doi.org/10.1055/s-0037-1610365.

\section{References}

(1) (a) Burja, A. M.; Banaigs, B.; Mansour, A. E.; Burgess, J. G.; Wright, P. C. Tetrahedron 2001, 57, 9347. (b) Clark, B. R.; Engene, N.; Teasdale, M. E.; Rowley, D. C.; Matainaho, T.; Valeriote, F. A.; Gerwick, W. H. J. Nat. Prod. 2008, 71, 1530.

(2) (a) Tan, L. T. Phytochemistry 2007, 68, 954. (b) Gerwick, W. H.; Coates, R. C.; Engene, N.; Gerwick, L. G.; Grindberg, R.; Jones, A.; Sorrels, C. Microbe 2008, 3, 277.

(3) Harrigan, G. G.; Luesch, H.; Yoshida, W. Y.; Moore, R. E.; Nagle, D. G.; Biggs, J.; Park, P. U.; Paul, V. J. Nat. Prod. 1999, 62, 464.

(4) Engene, N.; Choi, H.; Esquenazi, E.; Byrum, T.; Villa, F. A.; Cao, Z.; Murray, T. F.; Dorrestein, P. C.; Gerwick, L.; Gerwick, W. H.J. Nat. Prod. 2011, 74, 1737.

(5) Shiina, I.; Takasuna, Y.; Suzuki, R.; Oshiumi, H.; Komiyama, Y.; Hitomi, S.; Fukui, H. Org. Lett. 2006, 8, 5279. 
(6) (a) Calter, M. A.; Liao, W.; Struss, J. A. J. Org. Chem. 2001, 66, 7500. (b) Handa, M.; Scheidt, K. A.; Bossart, M.; Zheng, N.; Roush, W. R. J. Org. Chem. 2008, 73, 1031.

(7) (a) Evans, D. A.; Tedrow, J. S.; Shaw, J. T.; Downey, C. W. J. Am. Chem. Soc. 2002, 124, 392. (b) Evans, D. A.; Tedrow, J. S.; Shaw, J. T.; Downey, C. W. Org. Lett. 2002, 7, 1127. (c) Crimmins, M. T.; King, B. W.; Tabet, E. A.; Chaudhary, K. J. Org. Chem. 2001, 66,
894. (d) May, A. E.; Connell, N. T.; Dahlmann, H. A.; Hoye, T. R. Synlett 2010, 1984. (e) Botubol, J. M.; Sanchez, A. J. M.; Collado, I. G.; Galan, R. H. Eur. J. Org. Chem. 2013, 2420.

(8) (a) Paik, S.; Carmeli, S.; Cullingham, J.; Moore, R. E.; Patterson, G. M. L.; Tius, M. A. J. Am. Chem. Soc. 1994, 116, 8116. (b) Pearson, A. J.; Zhang, P. J. Org. Chem. 1996, 61, 9603.

(9) Wang, G.; Goyal, N.; Hopkinson, B. Bioorg. Med. Chem. Lett. 2009, 19, 3798. 